\title{
Double Trouble - Advanced Coronary Blood Flow Divertion: A Coronary Subclavian Steal Syndrome
}

\author{
Ryan D Andal ${ }^{1 *}$, Jasmin Melissa Bernardo ${ }^{2}$ and Joaquin Jison ${ }^{2}$ \\ ${ }^{1}$ Department of Cardiology, Makati Medical Center, Philippines \\ ${ }^{2}$ Department of Cardiology, Makati Medical Center, Philippines
}

Submission: May 16, 2017; Published: June 05, 2017

*Corresponding author: Ryan D Andal, Department of Cardiology, Makati Medical Center, Philippines, Email: ry_andal@yahoo.com

\begin{abstract}
Background: Coronary subclavian steal syndrome is a rare disorder, mostly unrecognized and under diagnosed, seen in patients developing subclavian artery stenosis after Coronary Artery Bypass Graft using the LIMA-LAD graft diverting blood flow from the native coronary LAD back to the graft LIMA-LAD into the subclavian artery in a retrograde fashion. This disorder causes significant symptoms which are brought about by ischemia coming from the heart, left upper extremity and the brain which was worsened by the ongoing dialysis.

Case Presentation: We describe a case of a 58-year-old male, with history of diabetes mellitus, hypertension , post CABG using LIMALAD graft and Mitral valve replacement 6 years ago, who developed Chronic Kidney Disease (CKD) requiring dialysis 3x a week using AV fistula left arm. 1 week prior, he developed grade 3-4/10 chest heaviness, dull in character with associated headache and left arm pain while having dialysis. Hemodialysis (HD) blood flow rate was reduced to decrease the symptoms. Until 30 minutes post HD now with severe intensity $10 / 10$ chest pain radiating to left shoulder with associated left hand numbness/weakness, headache and shortness of breath. Computed Tomography of the chest with contrast incidentally showed a short segment thrombus in the proximal left subclavian artery at its origin from the aortic arch, resulting in severe luminal stenosis or near total occlusion. Left heart catheterization with coronary angiography,selective angiogram of the left subclavian and right brachiocephalic artery showed the presence of a 90-95 percent occlusion at the proximal subclavian artery most probably due to a calcific plaque and or thrombus. Attempt was made to pass the guidewire through the occlusion but was unsuccessful. Since the lesion could not be percutaneously reperfused, decision to proceed to catheter directed thrombolysis was made with plans for surgery afterwards. A catheter directed streptokinase 250,000 IU was injected over 30 mins. Repeat selective angiography of the SA showed calcified left subclavian artery with $10 \%$ narrowing at the base with good arterial flow all the way to the left brachial artery. The vertebral artery and LIMA-LAD grafts were patent.
\end{abstract}

Conclusion: While the use of a LIMA graft with CABG has become widely accepted as the standard of care, left subclavian artery stenosis can compromise anterograde blood flow in the LIMA and if severe, can result in coronary subclavian steal syndrome. It is suggested that if there is a post CABG patient on dialysis, the presence of a supraclavicular bruit and an interarm BP difference of at least $20 \mathrm{mmhg}$ should be routinely done prior to dialysis detect early SA stenosis, especially if during dialysis they have symptoms of chest pain, vertebrobasilar insufficiency and limb ischemia would be valuable. Close follow-up of such patients is of utmost importance, as any future recurrent symptoms could be fatal due to the underlying functionally impaired conduit. Attention should be placed in considering site of HD access in post valve repair and post $\mathrm{CABG}$ requiring dialysis and if possible to perform also subclavian angiography whenever a post CABG patient on HD undergoes coronary angiogram.

Keywords: Coronary blood flow; Steal syndrome; Subclavian; Chronic Kidney Disease

Abbreviations: CKD: Chronic Kidney Disease; HD: Hemodialysis; SA: Subclavian Artery; CABG: Coronary Artery Bypass Graft; ECG: Electrocardiogram; CT: Computed Tomography

\section{Introduction (Figure 1) [1]}

Coronary subclavian steal syndrome is a rare disorder, mostly unrecognized and under diagnosed, seen in patients developing subclavian artery (SA) stenosis after Coronary Artery Bypass Graft (CABG) using the LIMA-LAD graft diverting blood flow from the native coronary LAD back to the graft LIMA-LAD into the subclavian artery in a retrograde fashion. This is also the same for the vertebral artery where blood is diverted back from the brain into the subclavian artery.

This disorder causes significant symptoms which are brought about by ischemia coming from the heart, left upper extremity and the brain. It is a catastrophic event where only very few case reports have been described and reported. 


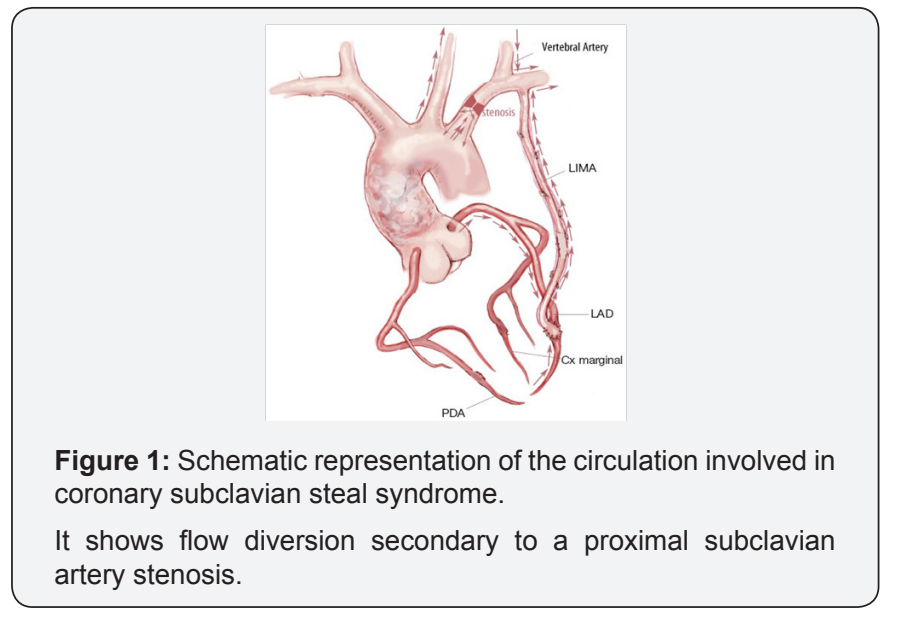

Moreover, it has been also described that a coronary steal from a LIMA graft post CABG can present as angina while undergoing hemodialysis. This phenomenon is documented through cardiac catheterization with evidence blood flow steal from the internal mammary artery graft while on dialysis, without subclavian artery stenosis.

This is a case report of a patient with coronary-subclavian steal syndrome caused by a proximal subclavian artery stenosis in a post CABG patient with a LIMA-LAD graft aggravated by ongoing dialysis using AV fistula on the left arm. A combination $f$ 2 phenomena that can lead to detrimental outcome if not treated appropriately though revascularization.

\section{Case Presentation}

We describe a case of a 58-year-old male, with history of diabetes mellitus, hypertension, post CABG using LIMA-LAD graft and Mitral valve replacement6 years ago, who developed Chronic Kidney Disease (CKD) requiring dialysis 3x a week, post AV fistula left arm a year ago. Since the bypass surgery, the patient had been doing well with no anginal symptoms and was maintained. 1 week prior, he developed grade 3-4/10 chest heaviness, dull in character with associated headache and left arm pain while having dialysis using the left AV fistula. Hemodialysis (HD) blood flow rate was reduced to decrease the symptoms. He was generally weak post HD with shortness of breath on exertion. The symptoms persisted everytime during HD. On the day od admission, symptoms recurred and persisted until 30 minutes post HD now with severe intensity 10/10 chest pain radiating to left shoulder with associated left hand numbness, headache and shortness of breath.

On physical examination the patient had an inter arm blood pressure difference of $110 \mathrm{mmHg}$ ( 40 palpatory BP in the left arm and 149/83 in right arm), cold pale looking left upper extremity with faint pulses and poor capillary refill, no AV fistula bruit, left , whereas the pulses in the lower extremities were normal. There was no supraclavicular bruit. Electrocardiogram (ECG) showed sinus rhythm with ST elevation at v2-v4, $1^{\text {st }}$ degree AV block (Figure 2).

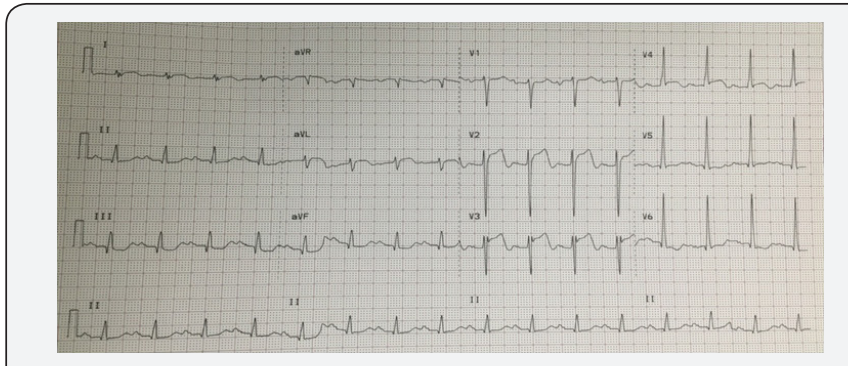

Figure 2: ECG tracing of the patient at the ER showing ST segment elevation.

Chest X-Ray showed pulmonary congestion and beginning edema. Troponin I was increased to $0.54 \mathrm{ng} / \mathrm{mL}$ (normal range $0.00-0.04 \mathrm{ng} / \mathrm{mL}$ ), increased CPKMB 20.7. ABG showed hypoxemia, He was initially diagnosed with anteroseptal wall ST elevation myocardial infarction but given the patients symptoms of chest pain with radiation to left shoulder and probable acute limb ischemia, a Computed Tomography (CT) of the chest with constrast was done to rule out aortic dissection (Figure 3).

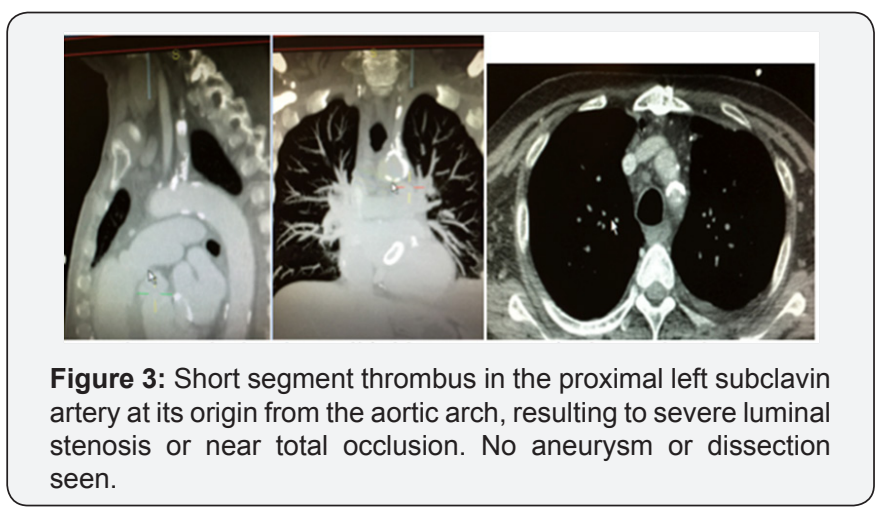

There was no aortic dissection seen in the study, however, the patient was incidentally noted to have a short segment thrombus in the proximal left subclavian artery at its origin from the aortic arch, resulting in severe luminal stenosis or near total occlusion. Immediate referral to Vascular Surgery was done.

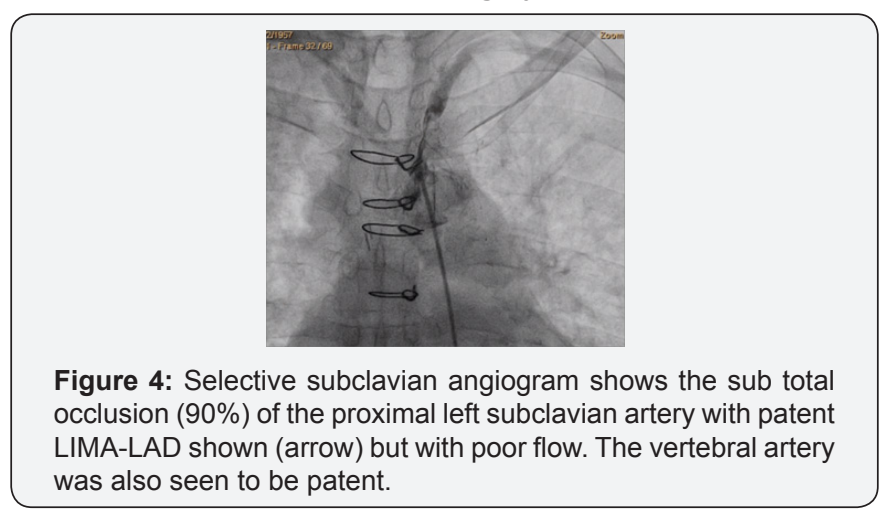

Left heart catheterization with coronary angiography, selective angiogram of the left subclavian and right brachiocephalic artery was performed. This confirmed the presence of a 90-95 percent occlusion at the proximal subclavian 
artery most probably due to a calcific plaque and or athrombus (Figure 4). Attempt was made to pass the guide wire through the occlusion but was unsuccessful. Since the lesion could not be percutaneously reperfused, decision to proceed to catheter directed thrombolysis was made with plans for surgery afterwards. Vascular access for hemodialysis via right intra jugular vein was inserted was made prior to thrombolysis.

A catheter directed streptokinase 250,000 IU was injected over 30mins. Repeat angiogram after streptokinase infusion showed persistence of the occlusion despite thrombolysis. Hemodialysis was initiated post angiogram. Surgical options were discussed: carotid-subclavian and subclavian artery bypasses; however, the patient declined surgical intervention due to the risks involved and financial considerations. There was slight improvement in the pulse, color and temperature of the left hand but the left AV fistula remains without bruit. Anticoagulation using enoxaparin and antiplatelets were given.

Repeat selective angiography of the SA showed calcified left subclavian artery with $10 \%$ narrowing at the base with good arterial flow all the way to the left brachial artery. The vertebral artery and LIMA-LAD grafts were patent (Figure $5 \& 6$ ).
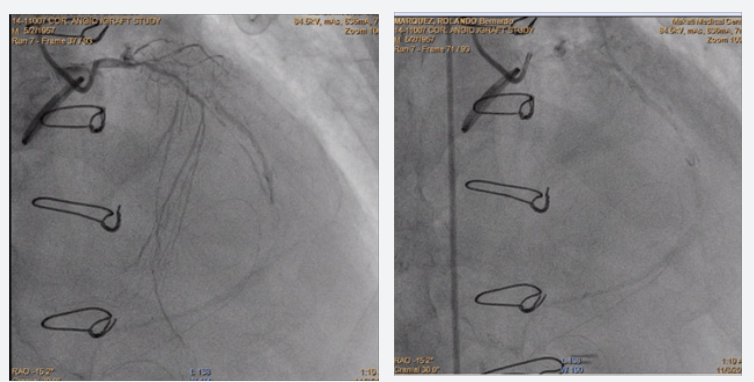

Figure 5: A. Left coronary angiography reveals severe stenosis of the LAD and circumflex artery. B. Three seconds interval delay showing retrograde flow up to the LIMA graft. (right anterior oblique projection). LAD, left anterior descending; LIMA, left internal mammary artery.

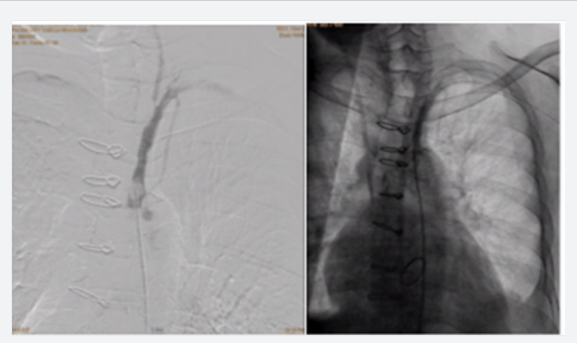

Figure 6: Repeat selective angiography of the subclavin artery showed calcified left subclavin artery with $10 \%$ narrowing at the base of subclavin artery with good arterial flow all the way to the left brachial artery. The vertebral artery and LIMA-LAD grafts were patient.

No further intervention was contemplated at this point. The stenosis did not warrant angioplasty nor any surgical considerations. Anticoagulation was continued for prevention of further thrombosis. Vascular access was relocated by creating a right AV fislula.

\section{Discussion}

Coronary subclavian steal syndrome is a rare cause of chest pain post CABG where myocardial ischemia is secondary to diversion of blood flow from the native coronary artery to the LIMA-LAD in a retrograde fashion supplying the more distal subclavian artery.

While the use of a LIMAgraft with CABG has become widely accepted as the standard of care, left subclavian artery stenosis can compromise anterograde blood flow in the LIMA and if severe, can result in coronary subclavian steal syndrome [2].

Ipsilateral subclavian artery stenosis is most commonly caused by atherosclerosis [3] or can be aggravated by other conditions such as vasculitic syndromes Takayasu arteritis [4], radiation arteritis [5] or from presence of hemodialysis AV fistula [6]. The presence of an AV fistula increases the blood flow rate going into the subclavian artery with preferential flow into the low resistance shunt. During dialysis the $(\mathrm{Qb})$ or blood flow requirement through the subclavian artery is magnified. Placement of AV access may result in increased cardiac output and increased cardiac oxygen demand in these patients. In the presence of a post CABG LIMA-LAD graft, it is documented though angiography of a retrograde flow through the LIMA-LAD during dialysis causing steals from the coronary arteries [6]. In this setting, the presence of a proximal subclavian artery stenosis would be catastrophic exhibiting a wide range of symptoms including neurologic, limb ischemia and coronary symptoms from the profound coronary subclavian steal syndrome during dialysis. The closure of the AV fistula left was able to help decrease the rate of steal effect.

SA stenosis /thrombosis in this patient has been associated with risk factors such as age, hypertension, diabetes mellitus, smoking, and nonsubclavian vascular calcification [7] have been implicated. Mitral valvular replacement and the presence of advanced atherosclerotic tendency of having dialysis using anipsilateral AV fistula has not been implicated. A history of peripheral vascular disease along with thepresence of an interarm blood pressure difference greater than $20 \mathrm{mmHg}$ has been proposed as clinical predictor of SA stenosis [8]. It is suggested that if there is a post CABG patient on dialysis, the presence of a supraclavicular bruit and an interatrial BP difference of at least $20 \mathrm{mmHg}$ should be routinely done prior to dialysis detect early SA stenosis, especially if during dialysis they have symptoms of chest pain, vertebrobasilar insufficiency including dizziness, syncope, ataxia, blurry vision, drop attacks and limb ischemia like upper extremity claudication and numbness would be valuable.

Definitive diagnosisis established with direct subclavian angiography, however routine performance of this test prior to consideration of coronary bypass surgery is ideal but impractical. 
Alternative is arterial duplex scanning of upper extremity or venous mapping. Current treatment options for coronary subclavian artery stenosis include endovascularor surgical revascularization. The endovascular approach with percutaneous transluminal angioplasty and peripheral stenting has been considered as first line treatment [9]. In our patient this was not an option due to inability to advance the guide wire to the stenosed area.

Coronary subclavian steal syndrome has been reported to be successfully managed via thoracotomy with carotid-subclavian, axillo-axillary bypass, and transposition of the subclavian artery up to the common carotid artery or even to the aorta $[10,11]$. Peri-operative mortality is low (0-0.8\%), and stroke rate ranges from $0.5-5 \%$. Five-year primary patency rates range from 92$95 \%$ and 8-10-year primary patency ranges from 83-95\% [10].

In severe stenosis without revascularization, there is limited data on outcomes with medical management. Finally, other causes of acute limb ischemia on top of subclavian stenosis lin in situ thrombosis or most especially cardioembolic cuases like intermittent atrial fibrillation should be thoroughly investigated and ruled out for these patients may require prolonged indefinite anticoagulant to prevent events.

Close follow-up of such patients is of utmost importance, as any future recurrent symptoms could be fatal due to the underlying functionally impaired conduit. Attention should be placed in considering site of HD access in post valve repair and post $C A B G$ requiring dialysis by carefully ruling out the presence subclavian artery stenosis by inter arm BP measurements and if possible to perform also subclavian angiography whenever a post CABG patient on HD undergoes coronary angiogram.

\section{References}

1. Younus U, Abbott B, Narasimha D, Page BJ (2014) Coronary Subclavian Steal Syndrome: An Unusual Cause of Angina in a Post-CABG Patient. Case Rep Cardiol 2014: 769273.

2. Cardon A, Leclercq C, Brenugat S, Jego P, Kerdiles Y (2002) Coronary subclavian steal syndrome after left internal mammarybypass in a patient with Takayasu's disease. J Cardiovasc Surg 43(4): 471-473.

3. Hull MC, Morris CG, Pepine CJ, Mendenhall NP (2003) Valvular dysfunction and carotid, subclavian, and coronaryartery disease in survivors of hodgkin lymphoma treated with radiation therapy. Journal of theAmericanMedical Association 290(21): 2831-2837.

4. Crowley SD, Butterly DW, Peter RH, Schwab SJ (2002) Coronary steal from a left internal mammary artery coronarybypass graft by a left upper extremity arteriovenous hemodialysis fistula. Am J Kidney Dis 40(4): 852-855.

5. Prasad A, Wassel CL, Jensky NE, Allison MA (2011) The epidemiology of subclavian artery calcification. J Vasc Surg 54(5): 1408-1413.

6. Osborn LA, Vernon SM, Reynolds B, Timm TC, Allen K (2002) Screening for subclavian artery stenosis in patients: whoare candidates for coronary bypass surgery. Catheterization and Cardiovascular Interventions 56(2): 162-165.

7. Henry M, Armor M, Henry I, Ethevenot G, Tzvetanov K, Chati Z (2007) Percutaneous transluminal angioplasty of the subclavian arteries. Int Angiol 26(4): 324-340.

8. Olsen CO, Dunton RF, Maggs PR, Lahey SJ (1988) Review of coronarysubclavian steal following internal mammary artery-coronary artery bypass surgery. Ann Thorac Surg 46: 675-678.

9. Chung DA, Large SR (2000) Relocation of the internal mammary artery graft in a case of coronary-subclavian steal. Thorac Cardiovasc Surg 48: $39-40$.

10. Bilku RA, Khogali SS, Been M (2003) Subclavian arterystenosis as a cause for recurrent angina after LIMA graft stenting. Heart 89(12): 1429.

11. Coronary artery bypass graft surgery, what is the state of art? Total arterial grafts.

\section{Your next submission with Juniper Publishers} will reach you the below assets

- Quality Editorial service

- Swift Peer Review

- Reprints availability

- E-prints Service

- Manuscript Podcast for convenient understanding

- Global attainment for your research

- Manuscript accessibility in different formats

( Pdf, E-pub, Full Text, Audio)

- Unceasing customer service

Track the below URL for one-step submission https://juniperpublishers.com/online-submission.php 